\title{
Point Cloud Data Processing and Analysis for 3D Measurement of Ship Hull Plate
}

\author{
Guiyang Deng1, Lianglun Cheng1, Xiaoqing Dong2* \\ ${ }^{1}$ School of Automation, Guangdong University of Technology, Guangzhou, Guangdong, China. \\ ${ }^{2}$ School of Physics and Electronic Engineering, Hanshan Normal University, Chaozhou , Guangdong, China. \\ * Corresponding author. Tel.: +860768-2317759; email: dxqzq110@163.com \\ Manuscript submitted February 4, 2019; accepted March 12, 2019. \\ doi: 10.17706/jsw.14.4.182-191
}

\begin{abstract}
In this paper, the 3D measurement of the hull plate is used as the background. It analyzes the principle of laser three-dimensional scanning. The independent k-neighbor problem is considered to improve the method of law loss propagation adjustment, at point cloud data segmentation. It improves the K-neighbor point cloud data boundary feature extraction algorithm. A point cloud reduction algorithm based on K-d tree space partitioning and local curvature threshold is proposed, and the algorithm flow is given. Finally, the related algorithms are simulated and tested, and the results also verify the feasibility of the above method, meet the needs of hull plate measurement.
\end{abstract}

Key words: Hull Plate, 3D Measurement, Point Cloud data processing, K-d tree.

\section{Introduction}

With the continuous development of information science and technology, three-dimensional simulation, physical reconstruction, virtual reality and other theories have been proposed.For the detection method of hull bending plate forming quality, we gradually change from the old plane two-dimensional space to the new space three-dimensional method(1-2).

The emergence of 3D laser scanner solves this practical problem. Through 3D laser scanning technology, also known as "real scene copy technology", its non-contact, fast scanning speed, large amount of information acquisition, high precision, real-time and full automation The advantages of complex environmental measurement, overcoming the limitations of traditional measuring instruments, become an important means to directly obtain high-precision three-dimensional data of the target and realize three-dimensional visualization(3).

The 3D laser scanning equipment of this paper adopts the product of American FARO company, the product model Focus3D, the appearance and environmental structure of the scanner measurement are shown in Fig. 1.

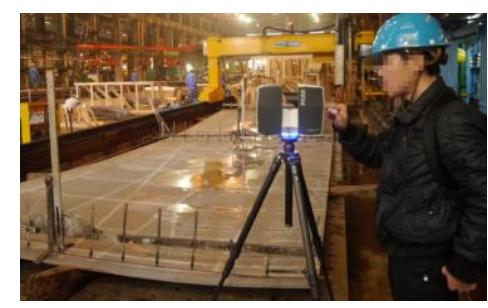

Fig.1. 3D laser scanner field data acquisition. 
The scanner realizes the measurement of the three-dimensional surface by means of the section line method. The section line method is to cut a set of curves by cutting a plane parallel to the surface to be measured, and first fix a section in the $\mathrm{X}$ axis before the actual measurement. The measurement is performed by moving one step at a certain step to obtain a data point on one section, that is, the scanning line; then, after moving a predetermined distance in the $\mathrm{Y}$ direction, the other section is successively moved in the $\mathrm{X}$ axis to obtain another section data, and finally The resulting data is a set of mutually parallel scan lines(1-3). The scanning path of the laser sensor during laser scanning is shown in Fig. 2.

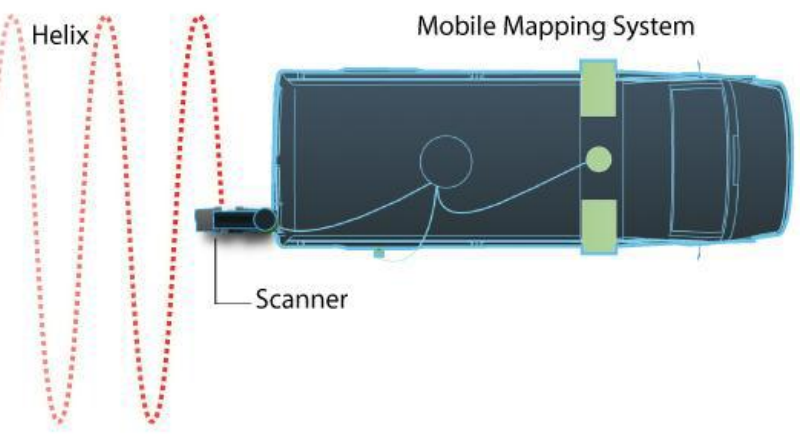

Fig. 2. Scanning path of the laser sensor.

\section{Point Cloud Data Processing}

Due to the complex curved surface of the hull plate, there are many types of curved surfaces, and the largest outer plate can reach more than 100 square meters.

The use of laser scanning equipment to obtain a large number of point clouds is quite large, and it is inevitably introduced With noise due to various factors such as the accuracy of the measuring equipment, the surface morphology of the ship to be tested, and the experience of the operator(3-4). Therefore, laser point cloud data processing is to obtain accurate and complete measurement data and to ensure the accuracy of the completion. Point cloud data processing mainly includes point cloud space segmentation, point cloud boundary feature information extraction, and point cloud data streamlining.

\subsection{Laser Scanning Measurement Principle}

The 3D laser scanner generally comprises a laser scanning module, a ranging module, an internal control module, a CCD (Charge-coupled Device) and a correction module. Two fast-rotating mirrors are built into the laser scanner. The narrow beam pulse of the laser emitter is reflected by the mirror to the object to be measured. The receiver receives the signal from the mirror and calculates the pulse from the transmission to the reception(5-6). The phase difference between the two can obtain the oblique intercept of the object to be tested to the scanner, and then calculate the three-dimensional coordinates of the laser spot on the object to be tested.

$$
\begin{aligned}
& X=S \cos \theta \cos \alpha \\
& Y=S \cos \theta \sin \alpha \\
& Z=S \sin \theta
\end{aligned}
$$

During the scan, set $\alpha$ is the scan lateral angle observation value, $\theta$ is the longitudinal angle observations. $\mathrm{S}$ is the oblique intercept of the measured object to the scanner, which is the return intensity of the scanning point. Laser scanning systems often use a custom coordinate system (as shown in Fig. 3), where the X-axis is 
in the lateral scanning plane, the $\mathrm{Y}$-axis is in the transverse scanning plane perpendicular to the $\mathrm{X}$-axis, and the Z-axis is perpendicular to the lateral scanning plane. The calculation formula of the three-dimensional coordinates on the object to be tested is as shown in the formula (1).

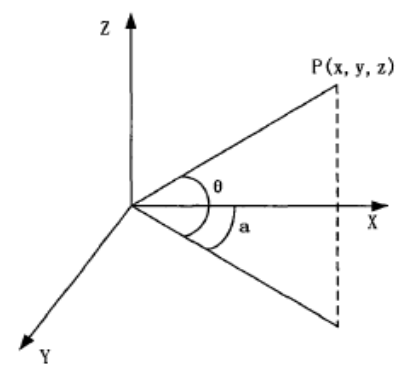

Fig. 3. Custom coordinate system.

\subsection{Point Cloud Space Segmentation}

The normal vector and curvature are important differential geometric properties that reflect the local geometric features of the surface, and are also the main influencing parameters of the point cloud space region. Before the point cloud space segmentation, the topological relationship of the scattered point cloud data is established, and the basic parameters such as the curvature and the normal vector at a certain point of the local surface are estimated(7-8). Aiming at the characteristics of scattered, disordered and no obvious topological relationship of curved point surface cloud data, the method of estimating local feature quantity is studied. Calculate the normal information of any point in the point cloud by fitting the micro-cut plane method; introduce the concept of curvature of the hypersurface feature, and according to the standard expansion of the surface at a certain point, the quadratic surface without cross terms, using quadratic surface The sample points in the neighborhood are combined to calculate the feature curvature in the three-dimensional space.

The steps to divide the point cloud data space are as follows:

First, read in the original point cloud data and find the minimum and maximum values of the point cloud data coordinates. $\left\{x_{\max }, x_{\min }, y_{\max }, y_{\min }, z_{\max }, z_{\min }\right\}$. Construct a large body bounding box that encloses all points, calculate the side length $\mathrm{L}$ of the sub-cube, and divide the minimum cuboid space of the point cloud data into $n_{\text {cube }}$.

$$
\left.n_{\text {cube }}=\left\lceil\left(x_{\max }-x_{\min }\right) / L \rrbracket\left(y_{\max }-y_{\min }\right) / L\right\rceil\left(z_{\max }-z_{\min }\right) / L\right\rceil
$$

where $\lceil$.$\rceil is rounded up. If the average number of data points in each subcube space is a function of \mathrm{k}$, then:

$$
n / n_{\text {cube }}=a k
$$

For the convenience of calculation, the formula (3) is substituted into the formula (2), and the calculation formula of the side length $L$ can be obtained after finishing:

$$
L=\beta \bullet \sqrt[3]{\frac{k}{n}\left(x_{\max }-x_{\min }\right)\left(y_{\text {max }}-y_{\text {min }}\right)\left(z_{\text {max }}-z_{\text {min }}\right)}
$$


where $\beta=\sqrt[3]{a}$ can adjust the side length value of the sub-cube, $\beta$ is the best value of $0.8 \sim 1.2$, and $\mathrm{n}$ is the total number of point clouds.

After the cube side length $L$ is determined, the minimum number of cube spaces in the xyz direction is:

$$
\left\{\begin{array}{l}
n_{x}=\left\lceil\left(x_{\text {max }}-x_{\text {min }}\right) / L\right\rceil \\
n_{y}=\left\lceil\left(y_{\text {max }}-y_{\text {min }}\right) / L\right\rceil \\
n_{z}=\left\lceil\left(z_{\text {max }}-z_{\text {min }}\right) / L\right\rceil
\end{array}\right.
$$

Finally, according to the size of the coordinate value, the scattered point cloud data is classified into different subspace cubes, and the space body not containing the data is deleted, which can reduce the number of subcube spaces searched. The point cloud data space segmentation effect is shown in Fig. 4.

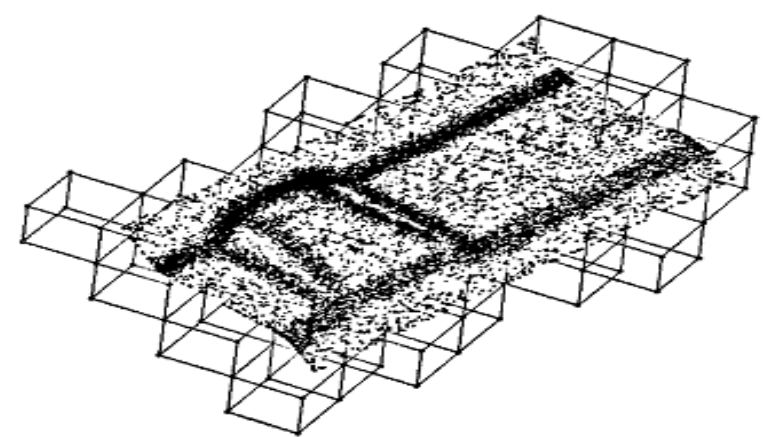

Fig. 4. Point cloud data space segmentation effect diagram.

In this paper, the micro-cut plane method is used to estimate the normal vector of scattered data points. Because of the inconsistency of the normal vector, it will affect the 3D reconstruction process and its follow-up. Therefore, the normal information must be adjusted. Su Xu proposed a method of normal vector propagation adjustment(5-9), adding a domain to the boundary point of the k-neighbor Riemannian graph $\cos t\left(\cos t=1-\left|n_{i} \bullet n_{j}\right|\right)$, and the threshold is a non-negative value.

When $\cos t$ tends to 0 , the two tangent planes will tend to be parallel, and the Riemannian graph is traversed to achieve the normal vector direction adjustment. This method needs to construct the propagation order, search all the data points, and the massive scattered point cloud will reduce the adjustment speed, increase the adjustment time, and the efficiency is extremely low. Meng Xianglin improved the minimum spanning tree method, and divided the scattered points into flat and non-flat points. Using the idea of propagation to adjust the normal vector, it is judged whether the neighborhood of the data points contains non-flat points to select the corresponding adjustment direction, and the adjustment method is improved(5-9). The efficiency of the vector direction, but neglecting the problem of independent k-neighborhood, this paper adopts the improved method of law loss propagation adjustment above, and adjusts the independent k-neighbor data to ensure the accuracy and fastness of normal adjustment. Sexuality can be used to generalize the normal vector information for the presence of unconnected data points.

\subsection{Point Cloud Boundary Feature Information Extraction}

The extraction boundary feature retention algorithm is the basis of constructing the reference plane and the local profile reference point set. Then, by comparing the distance from each point in the point set to the reference plane and the distance from the target point to the reference plane, the point cloud boundary feature is identified and stored. The specific process is as follows(10): 
By searching $\mathrm{k}$ neighbor points obtained by K-neighborhood, the local profile reference point set of candidate point $p$ is constructed as $X=\left\{x_{j} \mid j=0,1, \ldots ., k-1\right\}$ calculate the centroid $c\left(x_{i}, y_{i}, z_{i}\right)$ formula (6) of the reference point set

$$
x_{i}=k^{-1} \sum_{j=0}^{k-1} x_{j}, y_{i}=k^{-1} \sum_{j=0}^{k-1} y_{j}, z_{i}=k^{-1} \sum_{j=0}^{k-1} z_{j}
$$

Searching for a point $m_{i}$ farthest from the point $p$, calculating the distance $|p m|$ and the vector is perpendicular to the plane $\mathrm{L}$ of the local surface normal vector, and use it as a reference plane to observe the distribution of point sets. As shown in Fig. 5, set the coordinate of point $p$ be $\left(x_{p}, y_{p}, z_{p}\right)$ and the coordinate of point $c$ be $\left(x_{c}, y_{c}, z_{c}\right)$, then the equation of $\mathrm{L}$ on the reference plane can be expressed by equation (7):

$$
A x+B y+C z+D=0
$$

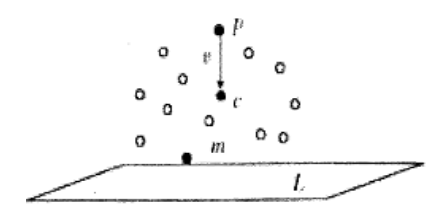

Fig. 5. Point set distribution status.

where $A=x_{p}-x_{c}, B=y_{p}-y_{c}, C=z_{p}-z_{c}, D$ expression is

$$
D=\left(x_{p}-x_{c}\right) x_{m}+\left(y_{p}-y_{c}\right) y_{m}+\left(z_{p}-z_{c}\right) z_{m}
$$

Let any point $X$ of the localized point set be, and its coordinate is $x=\left(x_{i}, y_{i}, z_{i}\right)$, then its distance to the plane $\mathrm{L}$ is calculated as:

$$
d_{(x, L)}=\left(A x_{i}+B y_{i}+C z_{i}+D\right)\left(A^{2}+B^{2}+C^{2}\right)^{\frac{1}{2}}
$$

Let $f(p)$ be the ratio of the maximum distance from point to plane $\mathrm{L}$ in point set $X$ and the distance from point $p$ to plane $\mathrm{L}$, with the probability that the feature $p$ is the boundary point, then the $f(p)$ formula is:

$$
f(p)=d(p, L)\left(\max \left\{d\left(x_{j}, L\right)\right\}\right)^{\frac{1}{2}}
$$

According to the given threshold $\sigma$ and $f(p)$, when $f(p)>\sigma$, then $p$ is the boundary point, otherwise $p$ is the internal point. In this paper, the data of multiple scattered point cloud data of different curved surface is tested. The range of 0.8-0.95 is suitable, but it will also be affected by various factors such as the density of data points obtained by different precision measuring equipment, and many features will be deleted by mistake. Point, the value of a given threshold will also change due to project specific requirements. The example proves that this method can better preserve the boundary feature point set of the point cloud.

\subsection{Point Cloud Data Streamlining}

Through the laser scanning equipment, the hull bending plate can be processed in a short time to process 
the curved surface point cloud data. Such dense point cloud data consumes a lot of resources and time in the process of preprocessing, storage, registration, transmission and reconstruction. reducing the algorithm execution efficiency and processing speed. Therefore, it is required to reduce the collected point cloud data while retaining the geometric features of the curved surface, reduce the amount of point cloud data processing, achieve efficient data processing, and achieve the goal of rapid reconstruction(11-12).

The streamlining of point cloud data can be processed and implemented in two stages. One is to adjust the projected optical strip image and the vertical sampling interval in the data acquisition phase, according to the deformation surface and resolution requirements of the curved surface of the curved panel. To determine the parameters of the data simplification, to achieve the first streamlined sampling data; second, after the point cloud data is collected and removed from the noise point, according to the actual engineering requirements, the corresponding algorithm is used to achieve data reduction(13).

For point data in flat areas, the bounding box method should be used to simplify point cloud data. Firstly, based on the kd tree space segmentation point cloud data, the k-neighborhood is used to calculate the neighboring data relationship in the local space where each leaf node is located, and the point cloud of the region is divided into sub-cubes with a side length of $L=1 \mathrm{~mm}$. Calculate the distance $d_{i}$ from a point $p_{i}$ in the subcube space to its center $O$. Let $o(x, y, z)$ be the center of a child node.

$$
x=\frac{x_{1}+x_{2}}{2}, y=\frac{y_{1}+y_{2}}{2}, y=\frac{y_{1}+y_{2}}{2}
$$

which is $O\left(x=\frac{x_{1}+x_{2}}{2}, y=\frac{y_{1}+y_{2}}{2}, y=\frac{y_{1}+y_{2}}{2}\right)$, any point in the child node $p_{i}\left(x_{i}, y_{i}, z_{i}\right)$ to the center distance:

$$
d_{i}=\sqrt{\left(x_{i}-x\right)^{2}+\left(y_{i}-y\right)^{2}+\left(z_{i}-z\right)^{2}}
$$

The formula (11) can be found on behalf of the person (12) to find $d_{i}$, compare $d_{i}$ to find the minimum distance $d_{\min }$ and retain the point corresponding to $d_{\min }$, delete other points in the subspace. The traversal of all the child nodes in the area in turn completes the data reduction of all bounding boxes. The point data of the rich detail area is reduced by the minimum distance method. The principle of minimum distance reduction is: first give a minimum distance $d_{\min }$ between two points, the distance between all points in the k-neighborhood $d_{i}$ is compared with $d_{\min }$ If $d_{i}<d_{\min }$, one of the two points will be deleted, otherwise two points will be retained; all the data points in the area will be judged in turn, and the point cloud is reduced.

\section{Algorithm Flow}

In this paper, a point cloud reduction algorithm based on the $\mathrm{k}-\mathrm{d}$ tree space partitioning and the curvature threshold of local surface features is proposed.

The K-d tree segmentation criterion is used to divide the 3D point cloud data into different hierarchical spaces, and the tree layer recursively forms a tree data model. In each node space, the K-neighbor domain calculation and the feature curvature estimation are used respectively to obtain the point cloud feature. The curvature information is set according to the curvature in the space of all the leaf nodes, and the adjustable curvature threshold is set, and the scattered point cloud data of the data source is divided into a relatively flat area and a richer detail area according to the threshold; and the space division is applied in the flat area.

The bounding box method completes the point cloud simplification to ensure the streamlined efficiency; 
the point cloud is simplified by the minimum distance method in the richer detail area, ensuring that the basic geometric information of the point cloud is not lost as much as possible, and the necessary feature information is retained for different types of surfaces. Streamline data and have high computational efficiency. The specific flow of the algorithm is shown in Fig. 6.

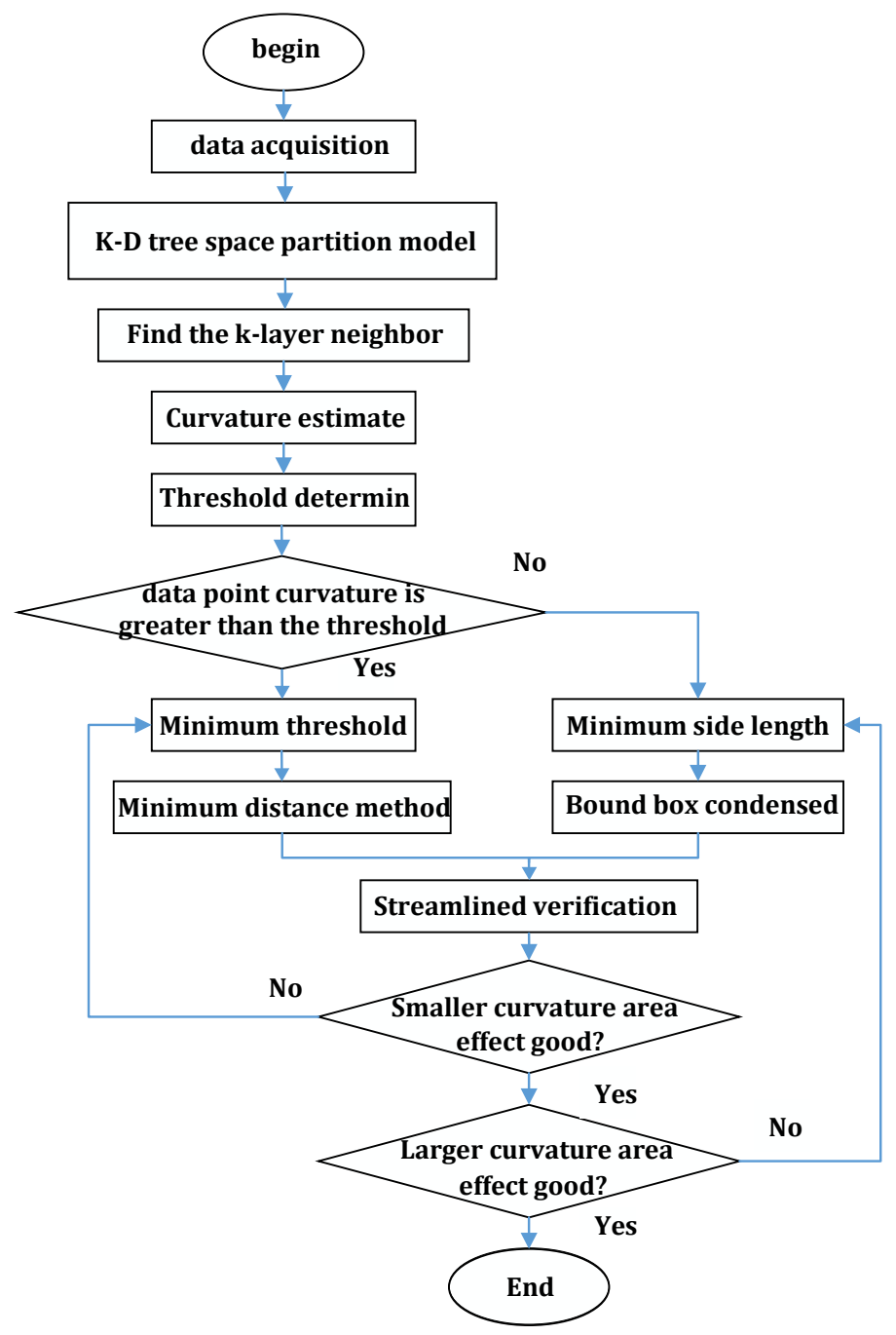

Fig. 6. Algorithm specific process.

\section{Experimental and Analysis}

According to the characteristics of the hull surface, We experimented and analyzed the laser scanning data of ship hull plate. Pixel Test Data of Partial Point Clouds as shown in Table 1. This paper selects the curvature change trend method to compress the data scale.

In the hull plate automatic processing machinery equipment jointly developed by the team and Guangzhou Shipyard International (as shown in Fig. 7), the method mentioned in this paper is carried out by using MATLAB. Simulation. Fig. 7 - Fig. 9 shows the front and back of the point cloud, and Fig. 7 shows the prototype of the curved surface of the hull. Fig. 9 shows the distribution of point cloud data processed by the algorithm. Fig. 9(b) is a simplified point cloud surface that is reconstructed. 
Table 1. Pixel Test Data of Partial Point Clouds (Unit: mm)

\begin{tabular}{llllllll}
\hline \hline Number & \multicolumn{2}{l}{ Pixel coordinates R } & \multicolumn{2}{l}{ Pixel coordinates L } & \multicolumn{2}{l}{ Three dimensional coordinates } \\
\hline 1 & 175 & 571 & 145 & 614 & 113.3582 & 2.4194 & 14.1774 \\
2 & 144 & 708 & 141 & 718 & 136.5365 & 0.7235 & 15.3425 \\
3 & 198 & 881 & 190 & 820 & 163.4527 & 8.3985 & 12.8767 \\
4 & 336 & 174 & 268 & 44 & 10.9025 & 26.9979 & -12.5576 \\
5 & 338 & 235 & 295 & 175 & 32.9894 & 29.5688 & -4.0785 \\
6 & 338 & 314 & 291 & 311 & 55.1245 & 29.2213 & 3.7438 \\
7 & 321 & 427 & 282 & 458 & 82.3542 & 26.9376 & 9.7359 \\
8 & 326 & 572 & 285 & 605 & 112.1466 & 27.2305 & 13.7965 \\
9 & 321 & 716 & 280 & 717 & 137.8663 & 25.9468 & 14.3945 \\
10 & 340 & 890 & 299 & 824 & 165.1278 & 28.3876 & 12.6562 \\
\hline \hline
\end{tabular}
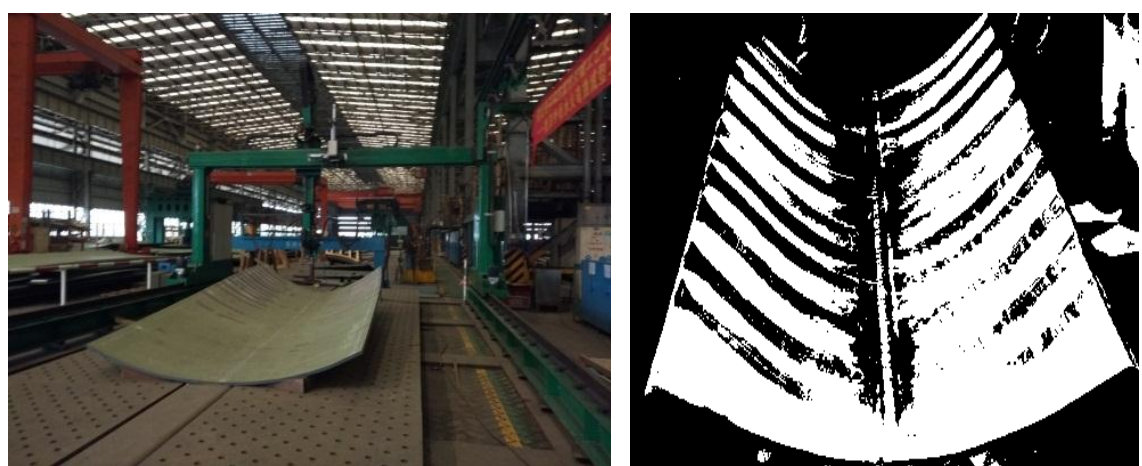

Fig. 7. (a) The hull plate automation robot (b) The original image.
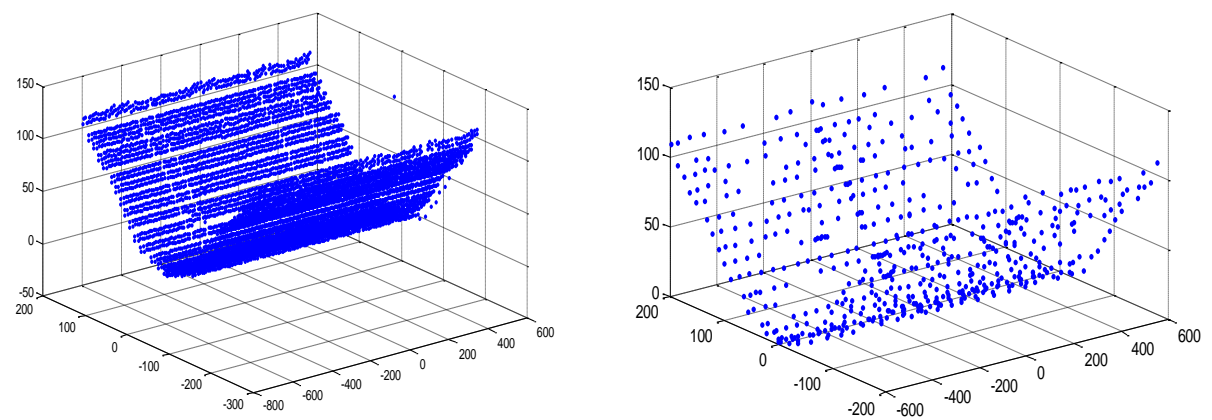

Fig. 8. (a)Minimum distance reduction method

(b) The bounding box method (unit: $\mathrm{mm}$ )
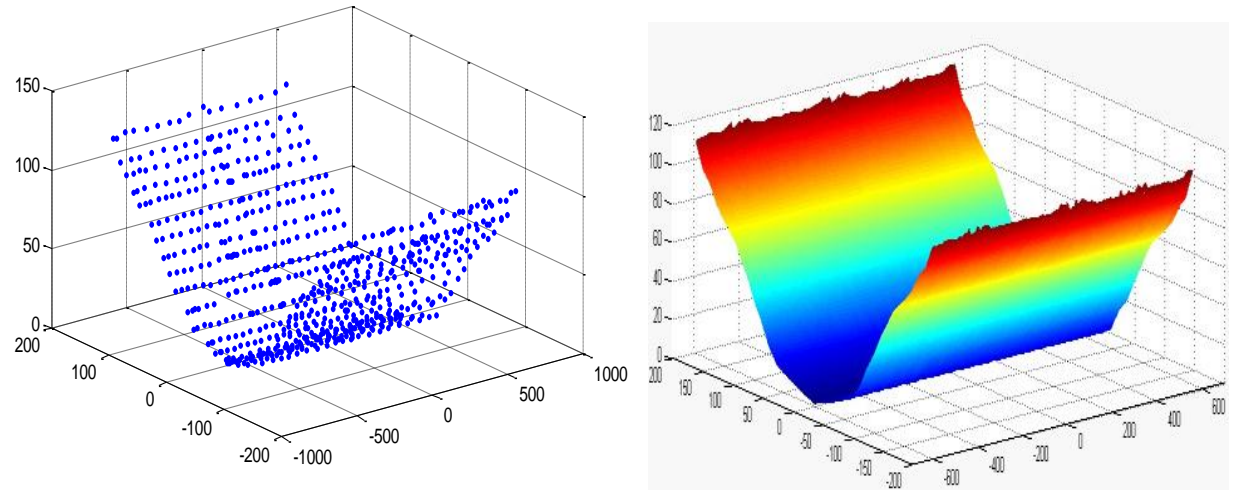

Fig.9. (a) The algorithm proposed in this paper

(b) Reconstruction of the point cloud surface (unit: $\mathrm{mm}$ ) 
The point cloud surface is reconstructed based on the feature curvature setting threshold segmentation streamlined point cloud. The algorithm is compared directly by using the bounding box method and the minimum distance method, as shown in Fig. 8(a) and Fig. 8(b). Among them, the bounding box method and the minimum distance parameter are basically the same during the experiment.

Table 2. Comparison of Algorithmic Streamlining Rates

\begin{tabular}{lll}
\hline \hline Algorithm & Points & Streamlining rate \\
\hline Original model & 3689274 & --- \\
Bound box method & 69524 & $98.16 \%$ \\
Minimum distance method & 3007891 & $18.47 \%$ \\
The algorithm proposed in this paper & 91472 & $97.52 \%$ \\
\hline \hline
\end{tabular}

Through laboratory analysis, as shown in Table 2: the minimum distance method can effectively preserve the surface features of the surface, but the reduction rate is too large, and the 3D reconstruction takes a lot of time; the bounding box method has a high simplification rate, but at the same time, a large number of feature points are lost, and high precision cannot be achieved. Reconstruct the curved surface; the algorithm of this paper combines the advantages of the two algorithms to better preserve the surface features and improve the simplification rate to some extent. Meet the needs of hull plate measurement.

\section{Conclusion}

This paper studies the extremely data processing problem of the three-dimensional measurement principle of the hull plate. Firstly, the calculation method of the three-dimensional coordinates of laser scanning is analyzed. Aiming at the point cloud space segmentation bounding box method, the method of adjusting the law loss propagation is improved and the corresponding k-neighbor data points are adjusted accordingly. Then the boundary feature extraction algorithm is constructed, the reference plane and the local profile reference point are set, the distance between each point in the point set and the target point to the reference plane is compared, and the point cloud boundary feature is identified and stored. In this paper, the point cloud is simplified by the K-d tree spatial hierarchy and feature curvature fusion algorithm, and the algorithm flow is given. Finally, a simulation experiment was carried out on the above algorithm. The results show that the proposed algorithm combines the advantages of the bounding box method and the minimum distance method to preserve the surface features and improve the simplification rate to some extent. Meet the needs of measuring the point cloud of the hull plate.

\section{Acknowledgment}

The author(s) disclosed receipt of the following financial support for the research, authorship, and/or publication of this article:

This work has been supported by the National Natural Science Foundation of China Guangdong Joint Funds(Grant No.U1701262 and No.U1801263) and partly supported by the Guangdong Provincial Key Laboratory of Cyber-Physical System(Grant No.2016B030301008) and the Guangdong applied science and technology R \& D special funds(Grant No. 2015B090922013) and the Guangdong Province Science and Technology Project(Grant No.2016B090918017) and the Guangdong NC First Generation Project(Grant No.2013B011302007) and the Guangzhou Science and Technology Project(Grant No.201604016107).

\section{References}

[1] Zhang, J., Hou, J., Wu, T., et al. (2018) Rapid surface reconstruction algorithm for 3D scattered point 
cloud model. Jisuanji Fuzhu Sheji Yu Tuxingxue Xuebao/Journal of Computer-Aided Design and Computer Graphics, 30(2), 235.

[2] Sun, S., Kuang, C., \& Xu, L. (2017). Three dimensional surface reconstruction based on light field imaging. Journal of Applied Optics.

[3] Galassi, F., Alkhalil, M., \& Lee, R., et al. (2018). 3D reconstruction of coronary arteries from 2D angiographic projections using non-uniform rational basis splines (NURBS) for accurate modelling of coronary stenoses. Plos One, 13(1).

[4] Hong, Q., Min, L., \& Qing, H. E., et al. (2016). Reconstruction of ship hull based on NURBS surface interpolation. Shipbuilding of China, 57(1),138-148.

[5] Chen, J. (2017). Application of ICP algorithm in TLS deformation monitoring. Modern Surveying \& Mapping.

[6] Bonnabel, S., Barczyk, M., \& Goulette, F. (2016). On the covariance of ICP-based scan-matching techniques. Proceedings of the American Control Conference.

[7] Chen, J., Liao, I. Y., \& Belaton, B., et al. (2015). A neural network-based point registration method for 3D rigid face image. World Wide Web-internet \& Web Information Systems, 18(2), 197-214.

[8] Lang, A., Song, Z., \& He, G., et al. (2017). Profile error evaluation of free-form surface using sequential quadratic programming algorithm. Precision Engineering, 47, 344-352.

[9] He, G., Huang, X., \& Guo, L., (2017) Evaluation of free-form surface profile error and analysis of uncertainty. Journal of Electronic Measurement \& Instrumentation.

[10] Seong, W. J., Ahn, J., \& Na, S. J., et al. (2010) Geometrical approach for flame forming of single curved ship hull plate. Journal of Materials Processing Technology, 210(13), 1811-1820.

[11] Tang, R., Zhang, B., \& Jin, G., et al. (2018) Multiple surface expansion method for design of freeform imaging systems. Optics Express, 26(3), 2983-2994.

[12] Qiu, L., Song, A., \& Sun, J., et al. (2017). A three-dimensional spiral smooth machining path based on the expansion surface. International Journal of Advanced Manufacturing Technology, 89(5-8), 1-7.

[13] Stupkiewicz, S. (2015). Extension of the node - to - segment contact element for surface - expansion dependent contact laws. International Journal for Numerical Methods in Engineering, 50(3), 739-759.

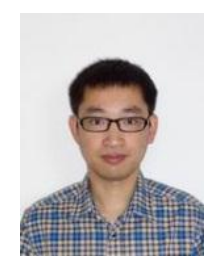

Guiyang Deng is a Ph.D. candidate in the Faculty of Automation, Guangdong University of Technology, China. He was born in Heilongjiang province, China, in 1981. He received his M.S.degree in electrical engineering and automation from Northeastern University, China in 2011. His current research interest is about digital image processing, Artificial intelligence and Industrial robot.

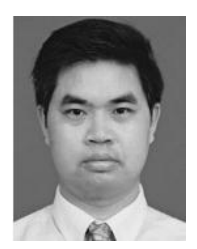

Lianglun Cheng is a professor and doctoral supervisor of Faculty of Automation, Guangdong University of Technology, China. He was born in Hubei province, China, in 1964. He received the PhD degree in Huazhong University of Science and Technology. His current research interest is about CPS, image data compression etc.

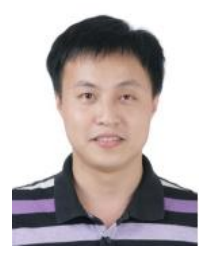

Xiaoqing Dong is an associate professor and master supervisor in Hanshan Normal University, China. He was born in Guangdong province, China, in 1982. He is now also a Ph.D. candidate of Guangdong University of Technology. He received his M.S.degree in signal and information processing from Guangdong University of Technology, China in June 2008. His current research interest includes wireless communication technology, internet of things and image processing. 